\title{
InGaN/GaN Nanowire LEDs and Lasers
}

\author{
Chao Zhao ${ }^{1}$, Tien Khee $\mathrm{Ng}^{1}$, Shafat Jahangir ${ }^{2}$, Thomas Frost ${ }^{2}$, Pallab Bhattacharya ${ }^{2}$, and Boon S. Ooi ${ }^{1, *}$ \\ ${ }^{1}$ Photonics Laboratory, King Abdullah University of Science and Technology, Thuwal 23955-6900, \\ Saudi Arabia \\ ${ }^{2}$ Center for Photonics and Multiscale Nanomaterials, Department of Electrical Engineering and \\ Computer Science, University of Michigan, Ann Arbor, Michigan 48109-2122, USA \\ *Email:boon.ooi@kaust.edu.sa
}

\begin{abstract}
The large specific surface, and the associated high density of surface states was found to limit the light output power and quantum efficiency of nanowire-array devices, despite their potential for addressing the "green-gap" and efficiency-droop issues. The phonon and carrier confinement in nanowires also led to junction heating, and reduced heat dissipation. In this paper, we will present our studies on effective surface states passivation in InGaN/GaN quantum-disks (Qdisks)-in-nanowire light-emitting diodes (LEDs) and lasers grown on silicon ( $\mathrm{Si}$ ), as well as our recent work on nanowires LEDs grown on bulk-metal, a non-conventional substrate.
\end{abstract}

Index Terms-InGaN/GaN quantum-disks-in-nanowire, light-emitting diodes, laser, Molecular Beam Epitaxy

\section{INTRODUCTION}

Group-III nitride based materials have been intensively studied for solid-state lighting and display applications. However, the performance of LEDs suffers from the lack of native substrates, high dislocation density, the existence of polarization fields, the related quantum-confined Stark effect (QCSE), and efficiency droop, etc. $[1,2]$.

Defect-free group-III nitride nanowires on silicon substrates, and green and red nanowire light-emitting diodes (NW-LEDs) have been developed in recent years, which demonstrated the potential of nanowires emitters for practical applications, beyond basic research. Compared to the planar LEDs, NW-LEDs were found to have reduced efficiency droop, reduced piezoelectric polarization field, and light extraction enhancement [3], which make them highly attractive visible light emitters. Yong-Ho Ra et al. reported coaxial InGaN/GaN quantum-well structures on $\mathrm{n}-\mathrm{GaN}$ core NW-LEDs without polarization [3]. Zetian Mi et al. demonstrated NW-LEDs with high internal quantum efficiency and nearly zero efficiency droop [4]. Moreover, InGaN/GaN NW-LEDs emitting at long wavelength and white light were also demonstrated $[5,6]$. For nanowire lasers, Chennupati Jagadish et al. reported lasing in optically pumped single nanowires made of GaAs and InP [7, 8], electrically-injected ultraviolet random lasing in AlGaN nanowires on Si has recently been demonstrated by Zetian Mi et al. [9]. Besides NW-LEDs, Pallab Bhattacharya et al. have demonstrated electrically pumped edge-emitting, green, red and near-infrared lasers using InGaN/GaN Qdisks in nanowires on silicon substrates. Previous integration of lasers and $\mathrm{Si}$ was achieved through wafer bonding [10], and growth of epitaxy on $\mathrm{Si}$ using buffer layers [11]. The nanowire lasers on $\mathrm{Si}$ we reported have performance surpassing or comparable to those of devices grown on $\mathrm{GaN}$, which can address various applications such as displays, solid state lighting, and silicon photonics [12-14].

Despite the achievement of high-quality nanowires and demonstration of nanowire devices, the high density of surface states was found to limit the output power and quantum efficiency of nanowire devices. It is noted that ammonium sulphide $\left(\mathrm{NH}_{4}\right)_{2} \mathrm{~S}_{x}$ has been commonly used for the surface passivation of III-V semiconductors. Organic sulfides, such as octadecylthiol (ODT), were used in passivating InAs materials [15]. Nevertheless, systematic studies of the passivation mechanism and the comparison of chemicals used for passivation of nitride nanowires and devices are required. Moreover, phonon and carrier confinement in these laterally discontinuous nanostructures led to reduced heat dissipation and increased junction heating. This requires further exploration into unconventional substrates.

In this paper, we will present our work on approach in surface states passivation in InGaN/GaN Qdisks in nanowire LEDs, as well as enhancing heat dissipation by growing high-quality nanowires on bulk-metal substrates.

\section{EXPERIMENTAL DETAILS}

The nanowires were prepared using Veeco plasma assisted molecular beam epitaxy (PAMBE) [6]. The NW-LEDs structures on Si have $\sim 300 \mathrm{~nm}$ n-type GaN $\left(n \sim 4 \times 10^{18} \mathrm{~cm}^{-3}\right), 8$ stacks of InGaN/GaN Qdisks and $\sim 150 \mathrm{~nm}$ p-type $\mathrm{GaN}(p \sim 7 \times$ $10^{17} \mathrm{~cm}^{-3}$ ) grown at $750{ }^{\circ} \mathrm{C}$. To study the surface passivation of NW-LEDs on Si, a sample was soaked in ODT solution for passivation before the LED fabrication. As for the Qdisks-in-nanowire LEDs on polycrystallinemolybdenum (Mo) substrate, the structure consists of $\sim 150 \mathrm{~nm}$ n-type $\mathrm{GaN}$ grown at $660{ }^{\circ} \mathrm{C}, 8$ stacks of InGaN-disks $(\sim 3$ $\mathrm{nm}) / \mathrm{GaN}$-barrier $(\sim 12 \mathrm{~nm})$ grown at $480{ }^{\circ} \mathrm{C}$ with both $\mathrm{Ga}$ and In fluxes of $3 \times 10^{-8}$ Torr. The $\mathrm{p}-\mathrm{GaN}(\sim 50 \mathrm{~nm})$ was grown at substrate temperature of $570{ }^{\circ} \mathrm{C}$, with Ga flux of $3.9 \times 10^{-8}$ Torr and $\mathrm{Mg}$ cell at $350^{\circ} \mathrm{C}$. Detailed NW-LEDs fabrication process can be found in our previous report [16].

\section{RESULTS AND DisCUSSION}

A. Enhanced surface passivation in nanowire LEDs for mitigating Shockley-Read-Hall (SRH) recombination

We treated the nanowires with ammonium sulfide to reduce the p-contact resistance of $\mathrm{GaN}: \mathrm{Mg}$ in the nanowire laser fabrication process [6], and further developed organic sulfide passivation process for LEDs. The passivation process results in the alteration of the surface dynamic charge, and recovered the band-edge emission, leading to a $\sim 4 \mathrm{X}$ increase in photoluminescence peak intensity, faster increasing trend in quantum efficiency and $50 \%$ increase in relative peak external-quantum-efficiency in these LEDs [17]. The is due to the reduction in SRH non-radiative recombination, a mono-molecular non-radiative recombination on the nanowires 
surface, confirmed using a novel 4D scanning ultrafast electron microscopy technique [18].
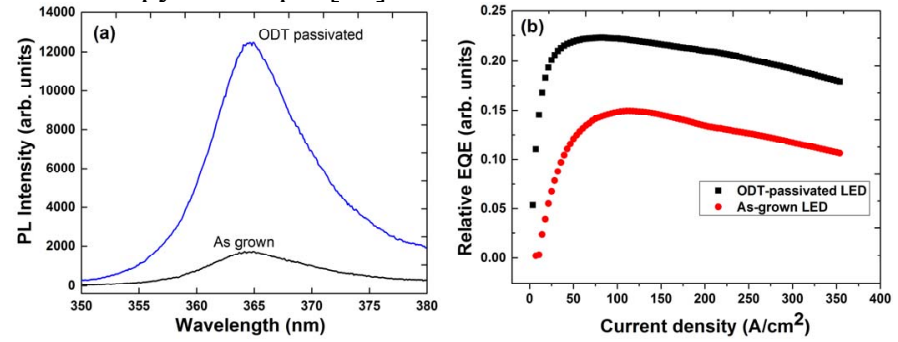

Fig. 1. (a) Room temperature GaN PL spectra of the sample without and with passivation; (b) the relative EQE of NW-LEDs without and with passivation.

\section{B. High-quality InGaN/GaN Qdisks-in-nanowires Bulk-Metal Substrates for High-power Light-emitters}

Current planar LEDs have to be transferred to a heat sink via wafer bonding or laser liftoff. In addition, nanowire LEDs on $\mathrm{Si}$ suffers from the formation of SiN layer during growth, and significant light absorption from $\mathrm{Si}$. We developed the first high-power red InGaN/GaN Qdisks-in-nanowire LEDs on Mo, which simultaneously implements buffer layer, n-metal contact, reflector and heat-sink, thus greatly simplifies the fabrication process [16]. The LEDs exhibited a low turn-on voltage of $\sim 2$ $\mathrm{V}$ without efficiency droop up to $1.6 \mathrm{kA} / \mathrm{cm}^{2}$. The light output power of $1.76 \mathrm{~mW}$ is higher than other reports on long wavelength $(705 \mathrm{~nm})$ LEDs, thus is promising for high-power device operation.
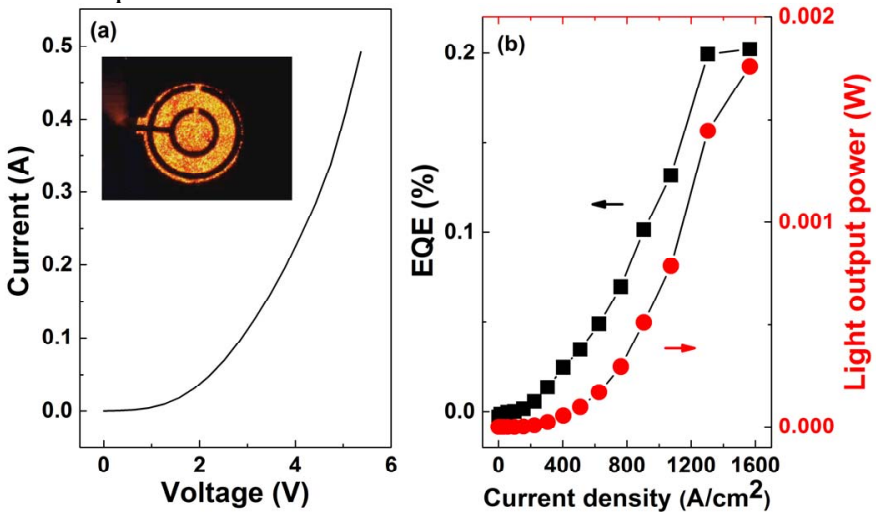

Fig. 2. (a) The measured $I-V$ characteristics of a $200-\mu \mathrm{m}$ diameter LED; the inset shows the optical microscope image of the NW-LED; (b) the EQE and L-I characteristics of the NW-LED.

\section{CONCLUSIONS}

In conclusion, we demonstrated an effective chemical passivation treatment on nitride nanowires, leading to the reduction of SRH non-radiative recombination. We also reported the first nanowires growth process on metal substrates. The InGaN/GaN Qdisks-in-nanowires grown on Mo exhibited high crystal quality, and the fabricated device shows considerably low turn-on voltage of $2 \mathrm{~V}$, achieves high-power, and emits at peak wavelength of $705 \mathrm{~nm}$. The InGaN/GaN nanowire lasers emitting at green-to-infrared wavelength range on Si were also demonstrated.

\section{ACKNOWLEDGMENT}

The authors acknowledge funding support from KAUST baseline funding (BAS/1/1614-01-01), King Abdulaziz City for Science and Technology (KACST) Technology Innovation Center (TIC) for Solid State Lighting (KACST TIC R2-FP-008).

\section{REFERENCES}

[1]S. Nakamura and G. Fasol, The blue laser diode: GaN based light emitters and lasers: Springer, 1997.

[2]J. Yan, J. Wang, Y. Zhang, P. Cong, L. Sun, Y. Tian, et al., "AlGaN-based deep-ultraviolet light-emitting diodes grown on High-quality AlN template using MOVPE," Journal of Crystal Growth, vol. 414, pp. 254-257, 2015.

[3]Y.-H. Ra, R. Navamathavan, J.-H. Park, and C.-R. Lee, "Coaxial InxGa1-xN/GaN Multiple Quantum Well Nanowire Arrays on Si(111) Substrate for High-Performance Light-Emitting Diodes," Nano Letters, vol. 13, pp. 3506-3516, 2013.

[4]N. Hieu Pham Trung, K. Cui, S. Zhang, M. Djavid, A. Korinek, G. A. Botton, et al., "Controlling Electron Overflow in Phosphor-Free InGaN/GaN Nanowire White Light-Emitting Diodes," Nano Letters, vol. 12, pp. 1317-1323, 2012.

[5] T. K. Ng, C. Zhao, C. Shen, S. Jahangir, B. Janjua, A. B. Slimane, et al., "Red to Near-Infrared Emission from InGaN/GaN Quantum-Disks-in-Nanowires LED," in CLEO: 2014, San Jose, California, 2014, p. SM2J.2.

[6]W. Guo, A. Banerjee, P. Bhattacharya, and B. S. Ooi, "InGaN/GaN disk-in-nanowire white light emitting diodes on (001) silicon," Applied Physics Letters, vol. 98, p. 193102, May 92011.

[7]D. Saxena, S. Mokkapati, P. Parkinson, N. Jiang, Q. Gao, H. H. Tan, et al., "Optically pumped room-temperature GaAs nanowire lasers," Nat Photon, vol. 7, pp. 963-968, 2013.

[8]Q. Gao, D. Saxena, F. Wang, L. Fu, S. Mokkapati, Y. Guo, et al., "Selective-Area Epitaxy of Pure Wurtzite InP Nanowires: High Quantum Efficiency and Room-Temperature Lasing," Nano Letters, vol. 14, pp. 5206-5211, 2014.

[9]K. H. Li, X. Liu, Q. Wang, S. Zhao, and Z. Mi, "Ultralow-threshold electrically injected $\mathrm{AlGaN}$ nanowire ultraviolet lasers on Si operating at low temperature," Nat Nano, vol. 10, pp. 140-144, 2015.

[10] K. Tanabe, K. Watanabe, and Y. Arakawa, "III-V/Si hybrid photonic devices by direct fusion bonding," Scientific Reports, vol. 2, p. 349, 04/02/online 2012.

[11] S. Chen, W. Li, J. Wu, Q. Jiang, M. Tang, S. Shutts, et al., "Electrically pumped continuous-wave III-V quantum dot lasers on silicon," Nat Photon, vol. advance online publication, 03/07/online 2016.

[12] T. Frost, S. Jahangir, E. Stark, S. Deshpande, A. Hazari, C. Zhao, et al., "Monolithic Electrically Injected Nanowire Array Edge-Emitting Laser on (001) Silicon," Nano Letters, vol. 14, pp. 4535-4541, 2014.

[13] S. Jahangir, T. Frost, A. Hazari, L. Yan, E. Stark, T. LaMountain, et al., "Small signal modulation characteristics of red-emitting $(\lambda=610 \mathrm{~nm})$ III-nitride nanowire array lasers on (001) silicon," Applied Physics Letters, vol. 106, p. $071108,2015$.

[14] A. Hazari, A. Aiello, T.-K. Ng, B. S. Ooi, and P. Bhattacharya, "III-nitride disk-in-nanowire $1.2 \mu \mathrm{m}$ monolithic diode laser on (001)silicon," Applied Physics Letters, vol. 107, p. 191107, 2015.

[15] M. H. Sun, H. J. Joyce, Q. Gao, H. H. Tan, C. Jagadish, and C. Z. Ning, "Removal of Surface States and Recovery of Band-Edge Emission in InAs Nanowires through Surface Passivation," Nano Letters, vol. 12, pp. 3378-3384, 2012.

[16] C. Zhao, T. K. Ng, N. Wei, A. Prabaswara, M. S. Alias, B. Janjua, et al., "Facile Formation of High-Quality InGaN/GaN Quantum-Disks-in-Nanowires on Bulk-Metal Substrates for High-Power Light-Emitters," Nano Letters, vol. 16, pp. 1056-1063, 2016.

[17] C. Zhao, T. K. Ng, A. Prabaswara, M. Conroy, S. Jahangir, T. Frost, et al., "An enhanced surface passivation effect in InGaN/GaN disk-in-nanowire light emitting diodes for mitigating Shockley-Read-Hall recombination," Nanoscale, vol. 7, pp. 16658-16665, 2015.

[18] J. I. Khan, A. Adhikari, J. Sun, D. Priante, R. Bose, B. S. Shaheen, et al., "Enhanced Optoelectronic Performance of a Passivated Nanowire-Based Device: Key Information from Real-Space Imaging Using 4D Electron Microscopy," Small, 2016. 